\title{
Structural Design of Wearable Lower Extremity Exoskeleton Based on the Human Body Engineering
}

\author{
Chunshan $\mathrm{He}^{1, \mathrm{a}}$, Liai Pan ${ }^{2, \mathrm{~b}}$, Qinghua $\mathrm{Li}^{3, \mathrm{c}}$ \\ ${ }^{1,2,3}$ College of Mechanical and Vehicle Engineering Changchun University, Changchun, 130022, \\ China \\ aemail: he03cs@126.com, 'email: panli636@126.com, email: zhitu@126.com
}

\begin{abstract}
Keywords: Lower Exoskeleton; Human Body Engineering; 3D Mdeling; Structural Design
\end{abstract}
\begin{abstract}
With the development of science and technology and the progress of the society, the wearable lower extremity exoskeleton robots become more and more popular. In the paper, on the basis of market research and the theory of ergonomics, design sizes of the lower extremity exoskeleton have been determined. Through analysis of the lower exoskeleton structure and movement, the structures of the wearable exoskeleton have been designed. And using Solidworks software, 3D model of the parts of exoskeleton has been drawn and the whole exoskeleton assembly has been completed; through mechanics analysis of the human body each joint, the stress of the three joints have been derived under the static and dynamic condition; Through the calculation of torque and power of each joint, it has been concluded that the maximum torque and maximum power driving the system were $150 \mathrm{Nm}$ and $150 \mathrm{~W}$, respectively.
\end{abstract}

\section{Introduction}

In our country, all kinds of people with disabilities are nearly 90 million, including nearly 30 million limb disabled people, the rest for intelligence, eyesight, hearing and other disabilities. Limb disabled people who need use artificial limbs are nearly 10 million, nearly 100 million people in osteoarthritis patients, more than 1 million patients with spinal cord injury. Thus the equipments demands of the auxiliary or rehabilitation training are great in China.

And with the development of science and technology and the progress of the society, the wearable lower extremity exoskeleton robots become more and more popular. It is belonging to been training robot and rehabilitation training robot is a branch of rehabilitation robots, is a kind of new robots appeared in recent years, is a category of medical robots [1]. At present the study of lower limb exoskeleton rehabilitation robot mainly included: In the mid1960s, American ge company cooperating with Cornell university jointly developed a called Hardiman (Human Augmentation Research and Development study) of the active control exoskeleton body; In the early 1990s, Japan Kanagawa engineering university successfully developed a wearable power coat; In 2000, BLEEX power robot has been developed in the university of California, Berkeley. [2][3][4] In 2002, a series of HAL wearable power robot have been developed in Tsukuba university Japan; In 2010, REX was developed in New Zealand REXBiotics company, it was the world's first hands-free, self-supporting and independent control system exoskeleton and so on. Domestic research started lately, and was still in laboratory research of universities and research institutes. The research was not only few and there is a great gap compared with foreign technology. Domestic research mainly included: walking Rehabilitation Training System (Gait Rehabilitation Training System, GRTS) has been developed in rehabilitation engineering research center, tsinghua University [5]. A multi degree of freedom of rehabilitation training robot has been developed in Zhejiang university, other universities were also in the positive research. Although some achievements have been made, but the complexity, flexibility and exoskeletons movement stability of the exoskeleton and exoskeleton control accuracy compared with the foreign has certain gap.

In the paper, on the basis of market research, the theory of ergonomics, modern mechanical design method, and structural design method of bionic personification, the structures of the wearable lower extremity exoskeleton have been designed to make it meet the use requirements of 
people.

\section{Determination of design sizes of the lower extremity exoskeleton}

Each part proportion of the human body is basically the same, according to the proportion, assuming that the wearer height $\mathrm{H}$, so:

Distance from the hip joint to foot for male: $0.045 \mathrm{H}+0.265 \mathrm{H}+0.471 \mathrm{H}=0.781 \mathrm{H}$, for female: $0.042 \mathrm{H}+0.261 \mathrm{H}+0.466 \mathrm{H}=0.769 \mathrm{H}$;

Distance from knee joint to the foot for male: $0.045 \mathrm{H}+0.265 \mathrm{H}=0.31 \mathrm{H}$, for female: $0.042 \mathrm{H}+0.261 \mathrm{H}=0.303 \mathrm{H}$;

Distance from the ankle joint to the foot for male: $0.045 \mathrm{H}$, for female: $0.042 \mathrm{H}$;

Waist size for male: $0.182 \mathrm{H}$, for female: $0.202 \mathrm{H}$.

Wearer's body are different, in order that the exoskeleton has good structure compatibility, the size of the thigh rod, calf rod and the belt were designed to the adjustable structure. Personal height is generally between $150 \mathrm{~cm}$ to $190 \mathrm{~cm}$, the maximum adjustable range of the structure was set to $20 \mathrm{~cm}$. So choose adult height $\mathrm{H}=170 \mathrm{~cm}$ for design size of each part of the exoskeleton. On the basis of the relevant ratio could be obtained:

The thigh length of the exoskeleton was $70 \mathrm{~cm} \sim 90 \mathrm{~cm}$;

The calf length of the exoskeleton was $40 \mathrm{~cm} \sim 52 \mathrm{~cm}$;

The waist size of the exoskeleton was $25 \mathrm{~cm} \sim 40 \mathrm{~cm}$.

\section{Material selection of the lower extremity exoskeleton}

Exoskeletons were worn on the lower limb of the human body, can support the body weight, and should not cause weight of the human body. For longer battery life, quality of exoskeleton should be light as possible under the premise meeting the stiffness and strength. Considering these factors, in the paper, the materials of exoskeleton was7075 aluminum alloy.It makes rehabilitation exoskeleton robot maintenance convenient, also more environmentally friendly. [7]

\section{Structural design of the wearable lower extremity exoskeleton}

In this paper, the l hip, knee, ankle, wear boots, belts and connecting rod of the exoskeleton have been designed.

\subsection{Structural design of the hip joint}

The hip joint of the body is typical ball - and - socket joints, bear the weight of the body, is also the most important joints of the body.

The wearable lower extremity exoskeleton robot was worn on the outside of human lower limb exoskeleton robot, so that there are space differences between the exoskeleton joint and the human body joint. When the exoskeleton mechanical leg drive the human body movement, the human body on the sagittal and coronal plane degree of freedom has certain error. [8] In this paper, with reference to design of BLEE exoskeleton hip joint, hip joint was designed to three degrees of freedom, namely, bending/stretching, outreach/adduction, spin in/spin out. [9] Bending/stretching was active degrees of freedom, and the rest were passive freedom; Outreach/adduction was designed on the back to take, spin in/spin out was designed on both sides of the body. On the flexion/above, both were the tandem.

In order to prevent mechanical legs moving range too big to injury human body lower limb when the rehabilitation training, limit switch have been designed. The limit range angle was same with the exoskeletons angle of the human body to ensure that the hip joint moved in the permission angle range. Belt back outreach/adduction place was aluminum alloy material, on both sides, the belt was the nylon, beam length of the exoskeleton could be adjusted. Structure of the hip joint was shown in figure 1. 


\subsection{Structural design of the knee joint}

The knee joint is the body of the more important joints, namely can bear body pressure, and is able to load and ease the shock, is the hub of lower limb activity. Freedom of knee joint of the exoskeleton is designed as flexor/active degrees of freedom, in order to prevent the exoskeleton due to excessive movement on the knee joint injury. The joints bend/extended range was limited to make the knee joint move in the allowed angle range, and ensure the safety of wearing training. Structure and limit device of the knee joint were shown in figure2.
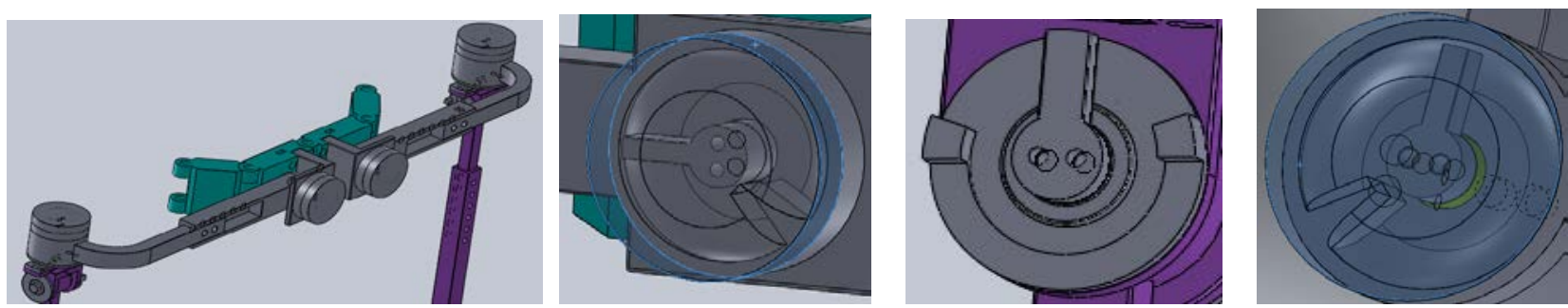

(a) General layout of hip (b) Outreach/adduction limit (c) Tracy/stretch limit (d) Spin in/ out limit

Fig.1. Structure and limit of the hip joint

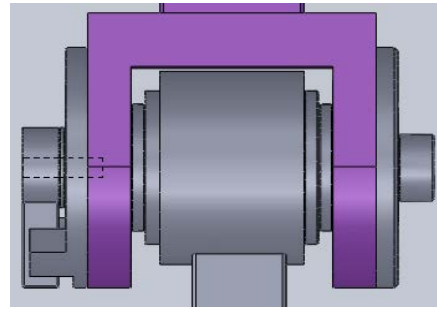

(a) Structure of the knee joint

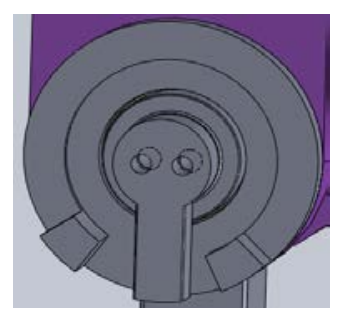

(b) Limit device of the knee joint

Fig.2. Structure and limit device of the knee joint

\subsection{Structural design of the ankle joint}

The ankle joint of the body has three degrees of freedom. When lower limb of the human moved, The ankle joint mainly completed the toe ankle flexion/dorsiflexion movement, the ankle joint outreach/adduction mainly regulated the movement direction of the lower limb, varus or valgus movement could adjust human body's balance when the road was uneven or feet met the impact. Structure and limit device of the ankle joint was shown in figure 3.

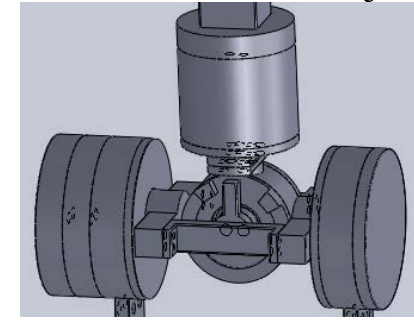

(a) Structure of the ankle joint

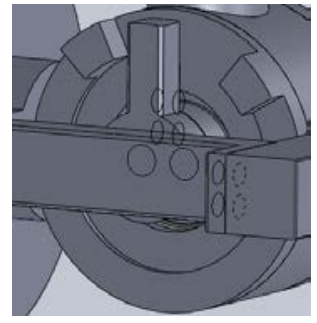

(b) Limit device of the ankle joint

Fig.3. Structure and limit device of the ankle joint

Size of the ankle joint was small. It was not easy to design such a complex joint structure in such a small size range. The ankle joint was designed to three degrees of freedom such as flexion/dorsiflexion, varus/valgus, spin in/spin out, the toe flexion/dorsiflexion was active joint degrees of freedom, the rest of the degrees of freedom were the passive joints.

\subsection{Structural design of the exoskeleton connecting rod}

Connecting rod of the lower exoskeleton robot was transmission mechanism and actuator mechanism. Connecting rod was very important to wear comfortable and effective rehabilitation training.

In order to make the exoskeleton widely use in lower limb movement disorder, the connecting rod was adjustable. If the length wasn't adjustable, it may cause misalignment between axis of hip, 
knee and ankle joint of the exoskeletons and axis of the wearer human lower bone joint. If both had deviation, movement interference has been appeared to be influent of rehabilitation training, may cause the wearer's lower limbs to harm. If it was serious, the exoskeleton would not be able to work. So it was very important that the exoskeleton connecting rod was adjustable.

In addition, because connecting rod should not only inherit the motor, the body weight, and need to be able to stand the weight of itself, the connecting rod should have enough carrying capacity; In order to prevent the connecting rod appearing too large deformation in the process of this movement and affect the exoskeleton wear safety and rehabilitation training, the connecting rod should have high stiffness, strength. Structure was shown in figure 4.

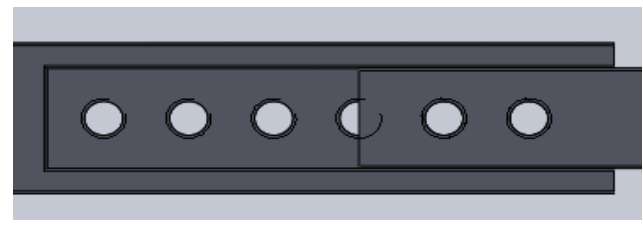

(a) The hip joint

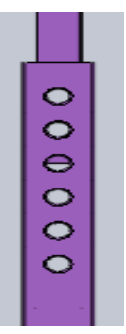

(b) The thig

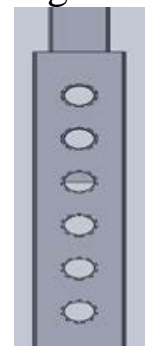

(c) The calf

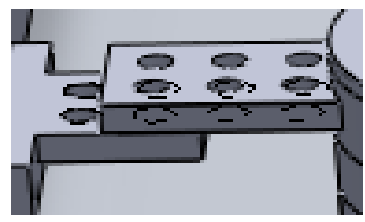

(d) The ankle

Fig.4. Structure of the adjustable connecting rod

\subsection{Structural design of wearing boots}

The main functions of foot are following (1) to maintain the body balance;(2) Boots created friction with the ground on the other hand to realize power forward movement. So when wearing boots were designed, the boots were designed hinge structure to imitate foot function of the human. Design structure of the wearing boots was shown in figure 5.

\subsection{Structural design of the belt}

The belt has a very important role to the lower wearable exoskeleton. It has not only the effect transferring between the two legs, but also the belt can fix exoskeleton joints. Through the wearing belt, the exoskeleton has been fixed worn on the body. Because the exoskeletons outreach/hip adduction degrees of freedom was on the back, material of the belt back of the exoskeleton was aluminum alloy, the outside was nylon material, and width of the belt was $60 \mathrm{~mm}$. To wear comfortable, the inside was with soft belt, structure of the belt was shown in figure 6 .

\subsection{Structural design of back frame}

The lower rehabilitation exoskeleton system included power drive system, control system, sensor system and executive system. Power battery and the control system design were fixed on the back frame, the control system was installed on the top of the back frame, and the power battery was installed at the bottom of the back frame. Back frame worn on human upper limb through belt. General assembly drawing of the lower rehabilitation exoskeleton was shown in figure 7.
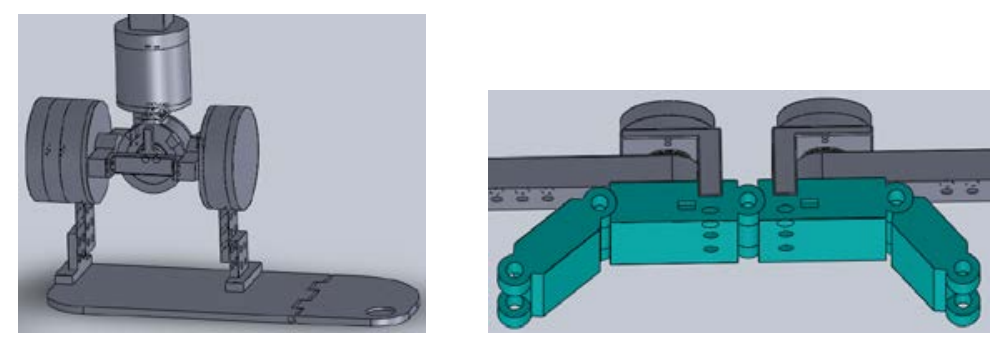

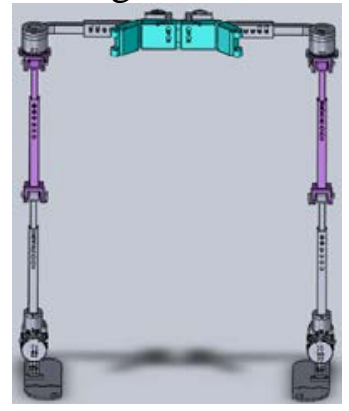

Fig.5. Wearing boots

Fig.6. Structure of the belt Fig.7. General assembly of lower exoskeleton 


\section{Mechanics analysis of the lower limb joint}

\subsection{Mechanics analysis of the hip joint}

Mechanics analysis of the hip joint mainly included static analysis and motion analysis.

(1) Static analysis of the hip joint

In this paper, Stresses of two kind of posture were analyzed that the human body standing with two legs and one leg.

1) Static analysis legs standing

When two legs stand, the gravity of the body was through the midline of the body, the hip joint didn't produce a dynamic torque. So on each of the femoral head, the force was $1 / 2$ of the body weight. And because weight of each lower limb was about 1/6 of the body weight, the force on each of the hip joint was the remaining two-thirds of 1/2, namely for a third of the weight.

2) Static analysis on one leg

Stand on one leg, weight what lateral femoral head bear was 4 times of the body weight. Human body on one foot could be seen as a similar lever structure, and lever fulcrum was femoral head. The ratio was about 1:3 to the arm from femoral head to the outside of the hip abductor muscle to the side of the pelvis with actuating arm, on both ends of the bearing ratio of 3:1. Condition of the force balance should be equal and opposite in all directions. Therefore, standing on one leg, the weight what the hip femoral head bear was about 4 times of the body weight.

(2) Force analysis of the hip joint when the lower limb moved

When the lower limb moved, force of the hip femoral was affected by acceleration on foot. Under normal pace, in stance phase follows, the force of the femoral head was about 5.8 times of the body weight.

In a gait cycle, the peak values of the two forces were on the femoral head. For male, a peak appears in the foot following the ground, the force was four times of the body weight; Another peak before when sufficient needle from the ground, the force could be up to 7 times of the body weight; During the foot flat, thanks to the rapid reduction of the body center of gravity, the joint force reduced to approximately equal to the human body weight; Lower limbs in during the swing phase, in order to make thigh to slow down, joint reaction force would be affected by extensor muscle contraction, its size was low and approximately equal to the body weight. For female, change pattern of the hip force basically was the same as male, the size was slightly low. [10]

\subsection{Mechanics analysis of the knee joint}

(1) Static analysis of the knee joint

Vertical standing in relaxation, centre of gravity of almost all body above knee was at the top of the patella joint center of rotation. Knee pressure was about 2 times of the body weight. Peak of the knee tolerance appeared in human lower limb knees $20^{\circ}$; mainly range of the endurance was in the range of human lower limb knees $10^{\circ}-40^{\circ}$.

(2) Force analysis of the knee joint when the lower limb moved

When the knee joint moved, the forces acting on the joints, the analysis on two factors must be considered: 1) the study of the acceleration of part of the body; 2) The mass inertia moment of the body. Walking, pressure what the knee joint bear was about $3 \sim 4$ times of the body weight, when the knee bended $90^{\circ}$, this force was up to $2.5 \sim 3$ times of the body weight.

\subsection{Mechanics analysis of the ankle joint}

When normal gait, because the feet provided forward momentum, and the force what ankles bear was greater than the force what the hip and knee bear. Full foot resting, feet flat standing load, the force on the ankle was about 2 times of the body weight; when foot was standing, the force what the ankle bear is about 3 times of the body weight; when walking, the force what the ankle bear was about five times of the body weight. The strength of the ankle joint determined the stability of the whole body as well as the stability of walking.

\subsection{Torque and power of each joint of the lower limbs}

(1)Position of mass and cancroids

Mass distribution and mass center position of each segment of human lower limbs were one of 
the important reference data of the mechanical design, such as connecting rod strength design, etc. Because of using the lower rehabilitation exoskeleton to take rehabilitation training, therefore, it was very important to dominate mass distribution of human lower limb. The joint transmission torque could be calculated. Based on the torque, the driving mode and power energy systems have been selected.

According to CT method, the relative mass distribution and its cancroids position of all parts of China adult human lower limbs were shown in table1.

Table.1 Relative mass distribution and cancroids position of parts of China adult human lower limbs

\begin{tabular}{|c|c|c|c|c|}
\hline \multirow{2}{*}{ Part } & \multicolumn{2}{|c|}{ Relative mass (\%) } & \multicolumn{2}{c|}{ (\%) Relative position } \\
\cline { 2 - 5 } & Male & Female & Male & Female \\
\hline The thigh & 28.38 & 28.20 & 45.3 & 44.2 \\
\hline The calf & 7.34 & 8.86 & 39.3 & 42.5 \\
\hline The foot & 2.96 & 2.48 & 48.6 & 54.9 \\
\hline
\end{tabular}

(2) Torque and power of each joint

According CGA database, in this paper, a body of normal, healthy, 28 years old, male was used as an example, its weight was $82 \mathrm{~kg}$, length of the leg was 0.99 meters, speed walking on flat ground was $1.27 \mathrm{~m} / \mathrm{s}$. torque and power variations of each joint of the lower limbs were shown in figure 8 .

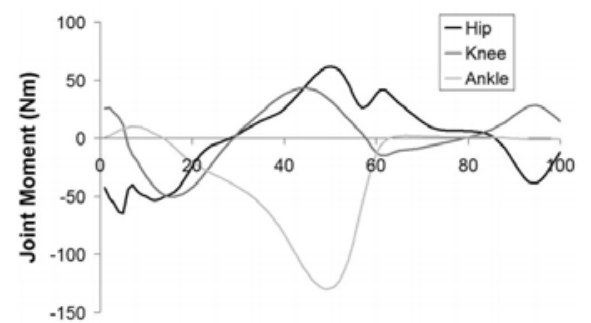

(a) Torque variation of hip, knee and ankle joints

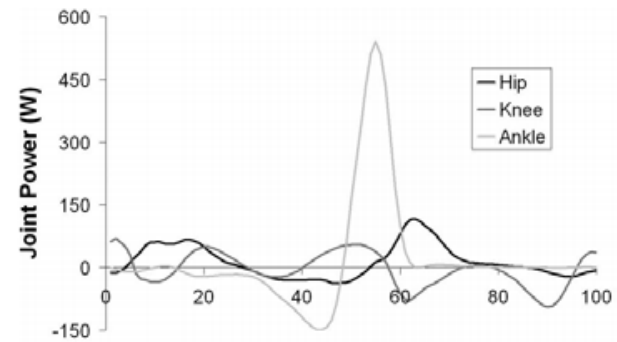

(b) Power variation of hip, knee and ankle joints

Fig.8. Torque and power variations of each joint of the lower limbs

The figure has shown that the joint of the maximum torque value was the ankle joints, which was about $150 \mathrm{Nm}$; the joint of the maximum power value was ankle joints of lower limb, which was about $580 \mathrm{~W}$. So, when choosing driving system of the lower limb rehabilitation exoskeleton, the maximum torque and maximum power what the driving system provided were $150 \mathrm{Nm}$ and $150 \mathrm{~W}$, respectively.

\section{Conclusion}

In the paper, on the basis of market research and the theory of ergonomics, design sizes of the lower extremity exoskeleton have been determined. Through analysis of the lower exoskeleton structure and movement, the structures of the wearable exoskeleton have been designed. And using Solidworks software, 3D model of the parts of exoskeleton has been drawn and the whole exoskeleton assembly has been completed; through the calculation of torque and power of each joint, it has been concluded that the maximum torque and maximum power driving the system were $150 \mathrm{Nm}$ and $150 \mathrm{~W}$, respectively.

\section{References}

[1] Feng Chen. Research of Wearable Power Robotics. [D] China University of Science and Technology. 2007

[2] Zoss,A.B.design of the Berkeley lower extremity exoskeleton(BLEEX) [J].Mechatronics, IEEE/ASME Transactions on.2006,11(2):128-138 
[3] Chu,A.Kazerooni,H.On the biomimetic design of the berkeley lower extremity exoskeleton (BLEEX)[C] //Robotics and Automation,2005.ICRA2005.Proceedings of the 2005 IEEE International Conference on. Barcelona,Spain:2005:4345-4352

[4] Zoss,A., Kazerooni, H. Design of an electrically actuated lower extremity exoskeleton[J]. Advanced Robotics.2006,20(9):967-988

[5] Jianan Li. Research Progress of Weight Training[J]. Chinese Journal of Physical Medicine and Rehabilitation, 2002,12,24(12):759-761

[6] Yongxiang Lu, Ying Chen. The man-machine integration system and technology[J] . Journal of Mechanical Engineering, 1994,30(6):1-9.

[7] Niankui Li etc. Aluminum alloy material and heat treatment technology[M]. Beijing: Metallurgical Industry Press,2012

[8] Yanjun Zhao. The working mechanism research of human lower limb exoskeleton[D]. Nanjing University of Science and Technology.2006

[9] Jing Jiang. Design and research of lower limbs powered exoskeleton mechanism[D] .Harbin Institute of Technology. 2012

[10] Jingzao Chen. Rehabilitation medicine[M].Beijing: Higher Education Press. 2007 